\title{
LPV SYSTEMS WITH BRIEF INSTABILITIES: APPLICATION TO INTEGRATED VISION/IMU NAVIGATION*
}

\author{
J. Hespanha ${ }^{\#}$, O. Yakimenko ${ }^{\&}$, I. Kaminer ${ }^{\&}$, A. Pascoal ${ }^{\dagger}+$ \\ \# University of Southern California, Los Angeles CA 90089, USA \\ fax: $\quad+1$ (213) 821-1109 \\ e-mail: hespanha@rcf-fs.usc.edu \\ \& Naval Postgraduate School, Monterey, CA 93943, USA \\ fax: $\quad+1$ (831) 656-2313 \\ e-mail: payakimeenps.navy.mil kaminerenps.navy.mil \\ † Institute Superior Técnico, Av. Rovisco Pais, 1096 Lisbon Codex, Portugal \\ fax: $\quad+351$ (1) 841-8291 \\ e-mail: antoniodisr.ist.utl.pt \\ * ECC2001 Conference \\ e-mail: ecc2001@fe.up.pt \\ http://www.fe.up.pt/ecc2001/
}

Keywords: linear parametrically varying systems, hybrid systems, autonomous vehicles, non-linear observers.

\begin{abstract}
This paper describes an application of recently developed results on linear parametrically varying systems (LPVs) with brief instabilities reported in [3] to the problem of designing a nonlinear vision/inertial navigation filter for an aircraft approaching an aircraft carrier is included. The results developed provide the proper framework to deal with out-of-frame events that arise when the vision system loses its target temporarily. Field tests with a prototype unmanned air vehicle illustrate the performance of the filter and illustrate the scope of applications of the new theory.
\end{abstract}

\section{Introduction}

In this paper we apply the results developed in [3] on the analysis of LPVs with brief instabilities to the design of the integrated vision/inertial filters. See [4] for an introduction to this problem and its application to the design of a navigation system for an aircraft approaching an aircraft carrier under the constraint that only passive sensors be used. The basic nonlinear filter structure adopted is described in [4], where the authors have obtained sufficient conditions for the existence of nonlinear integrated vision/inertial filters with guaranteed regional stability and performance. However, they did not address the fact that instabilities do occur when the vision system that is used to cancel the drift common to inertial sensors cannot be used temporarily because of out-of-frame events, i.e., periods of time when the vision system is unable to see the target due to occlusions or

* This work was supported by the Office of Naval Research under contract No. N0001497AF00002. the limited field of view. The results in [4] are extended in this paper to accommodate out-of-frame events.

The paper is organized as follows. Section 2 applies the theory developed in [3] to the design of an integrated vision/inertial filter. Section 3 includes description of the experimental setup used to test the filter performance. The paper ends with conclusions.

\section{Design of integrated vision/inertial filters}

In this section we apply the ideas formulated in [3] to the design of integrated vision/inertial filters. The basic filtering structure has been introduced in [4], where the authors obtained sufficient conditions for the existence of nonlinear integrated vision/inertial filters with guaranteed regional stability and performance. These results are extended in this paper to include so-called out-of-frame events.

\subsection{Problem formulation}

This section introduces the navigation problem that is the main focus of the paper and describes its mathematical formulation in terms of an equivalent filter design problem. For the sake of clarity, we first introduce some required notation and review the kinematic relationships of an aircraft / ship carrier ensemble, where the former is equipped with a vision based system.

Let $\{I\}$ denote an inertial reference frame, $\{B\}$ a body-fixed frame that moves with the aircraft, and $\{C\}$ a camera-fixed frame. The symbol $\{S\}$ denotes a ship-fixed body frame. The following symbols will be used:

- $\mathbf{p}_{B}=\left[\begin{array}{lll}x_{B} & y_{B} & z_{B}\end{array}\right]^{T}$ - position of the origin of $\{B\}$ measured in $\{I\}$ (i.e., inertial position of the aircraft);

- $\quad \mathbf{p}_{S}=\left[\begin{array}{lll}x_{S} & y_{S} & z_{S}\end{array}\right]^{T}$ - inertial position of the ship; 


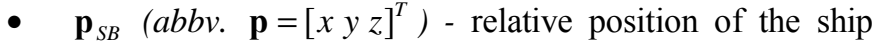
with respect to the aircraft, resolved in $\{T\}$;

- ${ }^{B} \mathbf{p}_{S B}$ (abbv. $\left.\mathbf{p}_{c}=\left[\begin{array}{lll}x_{c} & y_{c} z_{c}\end{array}\right]^{T}\right)$ - relative position of the ship with respect to the aircraft, resolved in $\{C\}$;

- $\quad \mathbf{v}_{B}$ - linear velocity of the origin of $\{B\}$ measured in $\{Z\}$ (i.e., inertial velocity of the aircraft);

- $\quad \mathbf{v}_{S}$ - inertial velocity of the ship;

- ${ }^{B} \mathbf{a}$ - linear acceleration of $\{B\}$ with respect to $\{I\}$, resolved in $\{B\}$;

- $\quad \omega$ - angular velocity of $\{C\}$ with respect to $\{I\}$, resolved in $\{I\}$;

- $\boldsymbol{\Lambda}=[\phi \theta \psi]^{T}$ - vector of roll, pitch, and yaw angles that parameterize locally the orientation of frame $\{C\}$ with respect to $\{I\}$.

Given two frames $\{A\}$ and $\{B\},{ }_{B}^{A} R$ denotes the rotation matrix from $\{B\}$ to $\{A\}$. In particular, ${ }_{C}^{I} R$ (abbreviated $R$ ) is the rotation matrix from $\{C\}$ to $\{I\}$, parameterized locally by $\Lambda$, that is, $R=R(\boldsymbol{\Lambda})$.

\subsection{Kinematic relations}

The rotation matrix $R$ satisfies the orthonormality condition $R^{T} R=I$. Furthermore, [1]:

$$
\dot{R}=R S(\omega),
$$

where

$$
S(\boldsymbol{\omega}):=\left[\begin{array}{ccc}
0 & -\omega_{z} & \omega_{y} \\
\omega_{z} & 0 & -\omega_{x} \\
-\omega_{y} & \omega_{x} & 0
\end{array}\right],
$$

is a skew symmetric matrix, that is, $S^{T}=-S$. The matrix $S$ satisfies the relationship $S(\mathbf{a}) \mathbf{b}=\mathbf{a} \times \mathbf{b}$, where $\mathbf{a}, \mathbf{b}$ are arbitrary vectors and $\times$ denotes the cross product operation. Furthermore, $\|S(\boldsymbol{\omega})\|=\|\boldsymbol{\omega}\|$.

We introduce the following assumption.

\section{$\mathbf{A 1}$ - The ship 's inertial velocity $\mathbf{v}_{S}$ is a non-zero constant.}

From the above definitions, it follows that

$$
\mathbf{p}_{S}=\mathbf{p}_{B}-{ }_{C}^{I} R \mathbf{p}_{C} \Rightarrow \frac{d^{2}}{d t^{2}}\left({ }_{C}^{I} R \mathbf{p}_{C}\right)=\frac{d^{2}}{d t^{2}} \mathbf{p}_{S}-\frac{d^{2}}{d t^{2}} \mathbf{p}_{B},
$$

and since $\frac{d^{2}}{d t^{2}} \mathbf{p}_{S}=0$ (assumption A1) we obtain

$$
\frac{d^{2}}{d t^{2}}\left({ }_{C}^{I} R \mathbf{p}_{C}\right)=-\frac{d^{2}}{d t^{2}} \mathbf{p}_{B} .
$$

Last equation shows that aside from a change in sign, the relative acceleration of the ship with respect to the aircraft resolved in $\{I\}$ is equal to the aircraft's inertial acceleration resolved in $\{I\}$.
However, in the case of strapdown inertial navigation systems widely in use today [8] the aircraft's inertial acceleration is usually given in $\{B\}$. Therefore, since

$$
\frac{d^{2}}{d t^{2}} \mathbf{p}_{B}={ }_{B}^{I} R^{B} \mathbf{a}
$$

it follows that

$$
\frac{d^{2}}{d t^{2}}\left({ }_{C}^{I} R \mathbf{p}_{C}\right)=-{ }_{B}^{I} R^{B} \mathbf{a}
$$

We assume that the image of the origin of $\{S\}$ acquired by a camera installed on-board the aircraft is obtained using a simple pinhole camera model of the form [2]

$$
\left[\begin{array}{l}
u \\
v
\end{array}\right]=\pi_{f}\left(x_{C}, y_{C}, z_{C}\right)=\frac{f}{x_{C}}\left[\begin{array}{l}
y_{C} \\
z_{C}
\end{array}\right] .
$$

where $f$ is the focal length of the camera and $[u v]^{T}$ are the image coordinates of $\mathbf{p}_{C}=\left[\begin{array}{lll}x_{C} & y_{C} & z_{C}\end{array}\right]^{T}$ in the camera's image plane. We also make the following assumption.

A2 $-x_{C}>0$, that is, the ship is always located in front of the camera's image plane.

We further assume that

A3 - the rotation matrices ${ }_{B}^{I} R$ and ${ }_{C}^{I} R$ are available from the onboard attitude measurement system.

This assumption is quite reasonable, considering the sophistication achieved by such systems today.

Suppose the aircraft is equipped with a barometric-based sensor that provides a measurement of the altitude of the aircraft with respect to the mean sea level. Then, using the relation $\mathbf{p}={ }_{C}^{I} R \mathbf{p}_{C}$ and assuming that the aircraft is sufficiently away from the ship (so as to neglect the height $h_{S}$ of the ship's deck above the mean sea surface), we may assume that

A4 - $h_{S}=0$.

Thus we obtain that the altitude measurement equals

$$
z=g_{\phi, \theta}\left(\mathbf{p}_{C}\right)=-\sin \theta x_{C}-\cos \theta \sin \phi y_{C}+\cos \theta \cos \phi z_{C} .
$$

where $\phi$ and $\theta$ are the roll and pitch angles in the rotation matrix ${ }_{C}^{I} R$.

We now introduce the underlying design model that plays a fundamental role in this paper. Let $\mathbf{y}=\left[\begin{array}{ll}u & v\end{array}\right]^{T}$. Then, the model that we consider can be written as

$$
G=\left\{\begin{array}{l}
\dot{\mathbf{p}}=\mathbf{v}, \\
\dot{\mathbf{v}}=-{ }_{B}^{I} R\left({ }^{B} \mathbf{a}_{m}+\mathbf{w}_{a}\right), \\
\mathbf{y}_{m}=g\left(\mathbf{p}_{C}\right)+\mathbf{w}_{y},
\end{array}\right.
$$

where $g\left(\mathbf{p}_{C}\right): \mathfrak{R}^{3} \rightarrow \mathfrak{R}^{3}$ is defined by 


$$
\left[\begin{array}{l}
u \\
v \\
z
\end{array}\right]=g\left(\mathbf{p}_{C}\right)=\left[\begin{array}{c}
\pi_{f}\left(\mathbf{p}_{C}\right) \\
g_{\phi, \theta}\left(\mathbf{p}_{C}\right)
\end{array}\right] .
$$

and $\mathbf{a}_{m}$ and $\mathbf{y}_{m}$ denote the measured values of $\mathbf{a}$ and $\mathbf{y}$, respectively, the measurements being corrupted by the process noises $\mathbf{w}_{a}$ and $\mathbf{w}_{y}$. In what follows, the deterministic set-up of $H_{\infty}$ filtering [6] will be adopted.

\subsection{Problem definition}

The problem that we consider in this paper consists of determining the relative position and relative velocity of an aircraft with respect to a landing site using vision and other on-board passive sensors. For the sake of clarity, we first tackle the simplified problem of designing a filter with no measurement noise in the model. This exercise is simple, yet it captures some of the key ideas used in the development that follows.

The additional notation that is required is introduced next. We let $\hat{\mathbf{p}}$ and $\hat{\mathbf{v}}$ denote estimates of $\mathbf{p}$ and $\mathbf{v}$, respectively. In the camera frame, they are denoted by $\hat{\mathbf{p}}_{C}, \hat{\mathbf{v}}_{C}$. We assume that the orientation of camera frame $\{S\}$ with respect to $\{I\}$ is restricted through the set

$\mathbf{A 5}-\boldsymbol{\Lambda}_{C}=\left\{\boldsymbol{\Lambda}:|\phi| \leq \phi_{\max },|\theta| \leq \theta_{\max },|\psi| \leq \psi_{\text {max }}\right\}$.

Notice, for example, that $\psi_{\max }$ should be set to $\pi$. We further assume that the vectors $\hat{\mathbf{p}}_{C}$ lie in the compact set

A6 - $P_{C}=\left\{\mathbf{p}_{C}: x_{\min } \leq x_{C} \leq x_{\max }, y_{\min } \leq y_{C} \leq y_{\max }\right.$, .

$$
\left.z_{\min } \leq z_{C} \leq z_{\max }\right\}^{\cdot}
$$

where $x_{\min }, \ldots, z_{\max }$ are determined from the geometry of the problem at hand. The set $P_{C}$ can be determined as follows. First, compute $P_{C}$ for a nominal orientation of the camera (usually inertial orientation). Determine the maximum range of camera orientation angles with respect to the nominal orientation. Then compute $P_{C}$ by allowing the angles to vary within these predetermined bounds.

In a realistic scenario the image of the ship smokestack will be lost by the onboard camera due, for example, to aircraft rotational motions. This phenomenon is known as an outof-frame event. Formally, we define a binary signal $s$ : $[0, \infty) \rightarrow\{0,1\}:$

$$
s(t):= \begin{cases}0 & \text { - out }- \text { of }- \text { frame event at time } t \\ 1 & \text { - camera tracks the smokestack at time } t .\end{cases}
$$

Furthermore, for a given binary signal $s$ and $t>\tau>0$, let us denote by $T_{s}(\tau, t)$ the amount of time in the interval $(\tau, t)$ that $s=0$. Formally,

$$
T_{s}(\tau, t):=\int_{\tau}^{t}(1-s(l)) d l,
$$

A7 - $s$ has brief out-of-frame event, i.e., $T_{s}(\tau, t) \leq T_{0}+\alpha(t-\tau), \quad \forall t \geq \tau \geq 0$, for some $T_{0} \geq 0$, $\alpha \in[0,1]$.

Navigation filter design will aim at ensuring that the estimates $\hat{p}_{C}$ of $\mathbf{p}_{C}$ lie in a compact set

$\hat{P}_{C}=\left\{\hat{\mathbf{p}}_{C}=\left[\begin{array}{lll}\hat{x}_{C} & \hat{y}_{C} \hat{z}_{C}\end{array}\right]^{T}:\left|\hat{x}_{C}-x_{C}\right| \leq x_{\max }-x_{\min }+d x\right.$,

$\left.\left|\hat{y}_{C}-y_{C}\right| \leq y_{\max }-y_{\min }+d y,\left|\hat{z}_{C}-z_{C}\right| \leq z_{\max }-z_{\min }+d z\right\}$

where $d x, d y$ and $d z$ are positive numbers, and $d x<x_{\min }$.

F1: Regional Stability. Consider the process model (1) and assume that $\mathbf{w}_{a}=\mathbf{w}_{y}=0$. For a given $\hat{P}_{C}$, find a number $\alpha_{0}>0$, and a dynamical system (filter) $F$ that operates on $\mathbf{y}_{m}$ and $\mathbf{a}_{m}$ to produce estimates $\hat{\mathbf{p}}$ of $\mathbf{p}$, and $\hat{\mathbf{v}}$ of $\mathbf{v}$ in the presence of out-of-frame events, such that

- $\hat{\mathbf{p}}_{C}(t) \in \hat{P}_{C}$ for any $t>0$,

- $\|\hat{\mathbf{p}}-\mathbf{p}\|+\|\hat{\mathbf{v}}-\mathbf{v}\| \rightarrow 0$ as $t \rightarrow \infty$,

provided that $\left\|\left(\hat{\mathbf{p}}_{C}(0)-\mathbf{p}_{C}(0), \hat{\mathbf{v}}(0)-\mathbf{v}(0)\right)^{T}\right\|<\alpha_{0}$.

Notice that the problem described aims at finding a filter that complements the information available from the vision system / barometric pressure sensor with that available from the inertial sensors.

The problem $\mathbf{F} 1$ focuses on the stability of the filter. The second filtering problem addresses the scenario where the performance of the filter in the presence of disturbances is considered.

F2: Regional Stability and Performance. Consider the process model (1) where $\mathbf{w}=\left[\mathbf{w}_{a} \mathbf{w}_{y}\right]^{T} \in L_{2},\|\mathbf{w}\|_{2} \leq \bar{\omega}$ and let the sets $P_{C}$ and $\hat{P}_{C}$ of allowable position vectors and allowable estimation vectors be defined as above. For given numbers $\gamma>0$ and $\alpha_{0}>0$, find a stable filter $F$ that operates on $\mathbf{y}_{m}$ and $\mathbf{a}_{m}$ to obtain estimates $\hat{\mathbf{p}}$ of $\mathbf{p}, \hat{\mathbf{v}}$ of $\mathbf{v}$ in the presence of out-of-frame events, such that if $\left\|\left[\left(\hat{\mathbf{p}}_{C}(0)-\mathbf{p}_{C}(0)\right)^{\mathrm{T}}(\hat{\mathbf{v}}(0)-\mathbf{v}(0))^{T}\right]^{T}\right\|<\alpha_{0}$, the filter satisfies the following conditions for all $\mathbf{w} \in L_{2}$ that $\|\mathbf{w}\|_{2} \leq \bar{\omega}$ :

- $\hat{\mathbf{p}}_{C}(t) \in \hat{P}_{C}$ for all $t \geq 0$,

- $\|\hat{\mathbf{p}}-\mathbf{p}\|+\|\hat{\mathbf{v}}-\mathbf{v}\| \rightarrow 0$ as $t \rightarrow \infty \bar{\square}$

${ }^{1}$ Given a signal $z$ we denote by $\|z\|_{2}$ the $L_{2}$-norm of $z$, i.e., $\|z\|_{2}=\sqrt{\int_{0}^{\infty}\|z(t)\|^{2} d t}$.

${ }^{2}$ As long as $\mathbf{w} \in L_{2}$ we always get convergence to zero. 
- $\quad\left\|T_{e w}\right\|_{2, i}<\gamma$, where $\mathbf{e}:=\hat{\mathbf{p}}-\mathbf{p}$ is the estimation error and $T_{e w}: \mathbf{w} \rightarrow \mathbf{e}$.

Notice the technical requirement that an allowable set of position estimates $\hat{P}_{C}$ be specified. As is shown later, this requirement is essential to establishing the boundedness of a certain operator for all possible values of the estimates $\hat{\mathbf{p}}$. In practice, the "size" of the allowable region $\hat{P}$ plays the role of a design parameter.

\subsection{Proposed solution}

This section describes the solutions to problems F1 and F2. First, however, we need the following basic results. Let $H$ denote the Jacobian of $g\left(\mathbf{p}_{C}\right)$ with respect to $\mathbf{p}_{C}$. From the definition of $g\left(\mathbf{p}_{C}\right)$, it follows that

$$
H\left(\mathbf{p}_{C}\right)=\left[\begin{array}{ccc}
-f y_{C} x_{C}^{-2} & f x_{C}^{-1} & 0 \\
-f z_{C} x_{C}^{-2} & 0 & f x_{C}^{-1} \\
-\sin \theta & -\cos \theta \sin \phi & \cos \theta \cos \phi
\end{array}\right] .
$$

It is easy to check that

$$
\operatorname{det}(H)=f^{2} x_{C}^{-3} z_{C}
$$

Therefore, $H$ is not invertible if and only if $z=0$. This implies that $H\left(\mathbf{p}_{C}\right)$ is invertible for all admissible values of $\mathbf{p}_{C}$, $\phi$, and $\theta$.

The following result provides a solution to problem $\mathbf{F 1}$.

Theorem 1 Let $\hat{P}_{C}$ be given and assume that A1-A7 hold and $r_{x}=\frac{x_{\max }-x_{\min }+d x}{x_{\min }}<1$. Suppose there exists a matrix $X=X^{T} \in \Re^{6 \times 6}$ and positive constants $\alpha, \alpha_{0}, \lambda_{0}, \mu, T_{0}$, such that $\alpha<\frac{\lambda_{0}}{\lambda_{0}+\mu}$ and

$$
\begin{aligned}
& X>0, \\
& F^{T} X+X F-2\left(1-r_{x}\right)^{2} \varepsilon C^{T} C \leq-\lambda_{0} X, \\
& F^{T} X+X F \leq \mu X, \\
& X-\delta^{-2} C^{T} C \geq 0, \\
& \alpha_{0}^{-2} e^{-\left(\lambda_{0}+\mu\right) T_{0}} I-X \geq 0,
\end{aligned}
$$

where $F:=\left[\begin{array}{ll}0 & I \\ 0 & 0\end{array}\right], C:=\left[\begin{array}{ll}I & 0\end{array}\right]$, and

$$
\begin{gathered}
\delta:=\min \left\{x_{\max }-x_{\min }+d x, y_{\max }-y_{\min }+d y, z_{\max }-z_{\min }+d z\right\}, \\
\varepsilon:=\min _{\hat{\mathbf{p}}_{C} \in \hat{P}_{C}} \lambda_{\min }\left(H^{T}\left(\hat{\mathbf{p}}_{C}\right) H\left(\hat{\mathbf{p}}_{C}\right)\right) .
\end{gathered}
$$

Define a filter (see Figure 1)

$$
F_{1}:=\left\{\begin{array}{l}
\dot{\hat{\mathbf{p}}}=\hat{\mathbf{v}}+s K_{1}{ }_{C}^{I} R H^{T}\left(\hat{\mathbf{p}}_{C}\right)\left(g\left(\hat{\mathbf{p}}_{C}\right)-\mathbf{y}_{m}\right), \\
\dot{\hat{\mathbf{v}}}=-{ }_{B}^{I} R^{B} \mathbf{a}_{m}+s K_{2}{ }_{C}^{I} R H^{T}\left(\hat{\mathbf{p}}_{C}\right)\left(g\left(\hat{\mathbf{p}}_{C}\right)-\mathbf{y}_{m}\right), \\
\hat{\mathbf{p}}_{C}={ }_{I}^{C} R \hat{\mathbf{p}},
\end{array}\right.
$$

where

$$
s(t):= \begin{cases}0 & - \text { out }- \text { of }- \text { frame event at time } t, \\ 1 & \text { - camera tracks the smokestack at time } t,\end{cases}
$$

and

$$
\left[\begin{array}{l}
K_{1} \\
K_{2}
\end{array}\right]:=-X^{-1}\left(1-r_{x}\right) C^{T} .
$$

Then the filter $F_{1}$ solves the filtering problem $\boldsymbol{F} \mathbf{1}$, provided that

$$
\left.\|\left(\hat{\mathbf{p}}_{C}(0)-\mathbf{p}_{C}(0)\right)^{\mathrm{T}}(\hat{\mathbf{v}}(0)-\mathbf{v}(0))^{T}\right]^{T} \| \leq \alpha_{0} .
$$

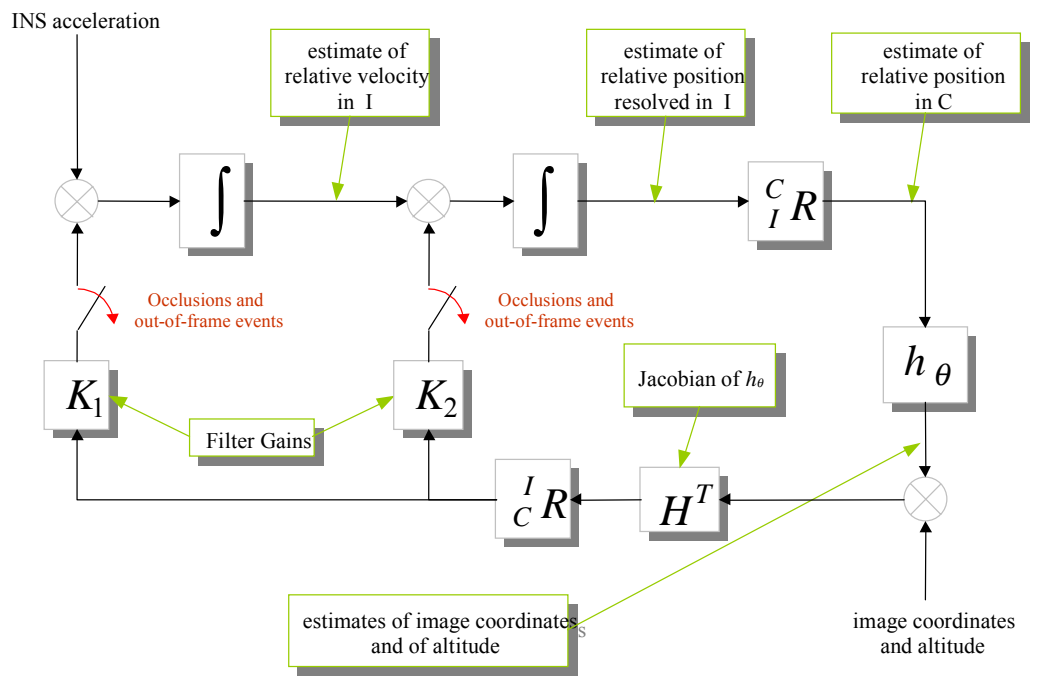

Figure 1: Filtering structure: filters $F_{1}$ and $F_{2}$. 
The solvability of the inequality (2) is addressed in [4]. There, it is shown that the inequality has a solution if and only if $r_{x}<1$. The next theorem provides a solution to the filtering problem $\mathbf{F} 2$.

Theorem 2 Let $\hat{P}_{C}$ be given and assume that A1-A7 hold and $r_{x}=\frac{x_{\max }-x_{\min }+d x}{x_{\min }}<1$. For a given gain $\gamma>0$, suppose there exists a matrix $X=X^{T} \in \mathfrak{R}^{6 \times 6}$ and positive constants $\alpha$, $\alpha_{0}, \lambda_{0}, \mu, T_{0}$, such that $\alpha<\frac{\lambda_{0}}{\lambda_{0}+\mu}$ and

$$
\begin{aligned}
& X>0 \\
& {\left[\begin{array}{cc}
F^{T} X+X F+\lambda_{0} X+\left(-\left(1-r_{x}\right)^{2} \varepsilon\right) C^{T} C & X F^{T} \\
F X & -I
\end{array}\right] \leq 0,} \\
& F^{T} X+X F+X F^{T} F X+\frac{e^{\left(\lambda_{0}+\mu\right) T_{0}}}{\lambda \gamma^{2}} C^{T} C \leq \mu X \\
& X-\delta^{-2} C^{T} C \geq 0 \\
& \alpha_{0}^{-2}\left(e^{-\left(\lambda_{0}+\mu\right) T_{0}}-\bar{\omega}^{2}\right) I-X \geq 0
\end{aligned}
$$

where $F:=\left[\begin{array}{ll}0 & I \\ 0 & 0\end{array}\right], C:=\left[\begin{array}{ll}I & 0\end{array}\right]$,

$\delta:=\min \left\{x_{\max }-x_{\text {min }}+d x, y_{\text {max }}-y_{\text {min }}+d y, z_{\text {max }}-z_{\text {min }}+d z\right\}$, and $\varepsilon:=\min _{\hat{\mathbf{p}}_{C} \in \hat{P}_{C}} \lambda_{\min }\left(\varphi_{1}\left(\hat{\mathbf{p}}_{C}\right)\right)$.

Define a filter (see Figure 1)

$$
F_{2}:=\left\{\begin{array}{l}
\dot{\hat{\mathbf{p}}}=\hat{\mathbf{v}}+s K_{1 C}{ }_{C}^{I} R H^{T}\left(\hat{\mathbf{p}}_{C}\right)\left(g\left(\hat{\mathbf{p}}_{C}\right)-\mathbf{y}_{m}\right), \\
\dot{\hat{\mathbf{v}}}=-{ }_{B}^{I} R^{B} \mathbf{a}_{m}+s K_{2 C}{ }_{C}^{I} R H^{T}\left(\hat{\mathbf{p}}_{C}\right)\left(g\left(\hat{\mathbf{p}}_{C}\right)-\mathbf{y}_{m}\right), \\
\hat{\mathbf{p}}_{C}={ }_{I}^{C} R \hat{\mathbf{p}},
\end{array}\right.
$$

where

$$
\left[\begin{array}{l}
K_{1} \\
K_{2}
\end{array}\right]:=-X^{-1}\left(1-r_{x}\right) C^{T}
$$

Then the filter $F_{2}$ solves the filtering problem $\mathbf{F} 2$ if

$$
\left\|\left[\left(\hat{\mathbf{p}}_{C}(0)-\mathbf{p}_{C}(0)\right)^{\mathrm{T}}(\hat{\mathbf{v}}(0)-\mathbf{v}(0))^{T}\right]^{T}\right\| \leq \alpha_{0} .
$$

The next theorem derives necessary and sufficient conditions under which LMI (3) is satisfied.

Theorem 3 Let $F, \gamma$ and $\varepsilon$ and be defined in Theorem 2. Then $\exists X=X^{T}>0$ such that

$$
\left[\begin{array}{cc}
F^{T} X+X F+\lambda_{0} X+\left(\frac{e^{\left(\lambda_{0}+\mu\right) T_{0}} \lambda_{0}}{\lambda \gamma^{2}} I-\left(1-r_{x}\right)^{2} \varepsilon\right) C^{T} C & X F^{T} \\
F X & -I
\end{array}\right] \leq 0
$$

$$
\Leftrightarrow \frac{e^{\left(\lambda_{0}+\mu\right) T_{0}} \lambda_{0}}{\lambda \gamma^{2}}-\left(1-r_{x}\right)^{2} \varepsilon \leq 0
$$

Remark Theorem 3 shows that the linear matrix inequality (LMI) (3) is feasible if and only if

$$
\frac{e^{\left(\lambda_{0}+\mu\right) T_{0}} \lambda_{0}}{\lambda \gamma^{2}} I-\left(1-r_{x}\right)^{2} \varepsilon \leq 0 \Leftrightarrow \gamma^{2} \geq \frac{e^{\left(\lambda_{0}+\mu\right) T_{0}} \lambda_{0}}{\lambda\left(1-r_{x}\right)^{2} \varepsilon} .
$$

Recall that

$$
\begin{array}{r}
\varepsilon=\min _{\hat{\mathbf{p}}_{C} \in \hat{P}_{C}}\left\{\| \lambda_{\min }\left(H^{T}\left(\hat{\mathbf{p}}_{C}\right) H_{T}\left(\hat{\mathbf{p}}_{C}\right)\right) \mid\right\} \\
\leq \min _{\mathbf{p}_{C} \in P_{C}}\left\{\mid \lambda_{\min }\left(H^{T}\left(\mathbf{p}_{C}\right) H_{T}\left(\mathbf{p}_{C}\right)\right) \|\right\}=\max _{\mathbf{p}_{C} \in P_{C}}\left\{\mid\left(H^{T}\left(\mathbf{p}_{C}\right) H_{T}\left(\mathbf{p}_{C}\right)\right)^{-1} \|\right\}^{-1}
\end{array}
$$

Therefore, we obtain

$$
\gamma^{2} \geq \frac{e^{\left(\lambda_{0}+\mu\right) T_{0}} \lambda_{0}}{\lambda\left(1-r_{x}\right)^{2}}=\max _{\mathbf{p}_{C} \in P_{C}}\left\{\left(H^{T}\left(\mathbf{p}_{C}\right) H_{T}\left(\mathbf{p}_{C}\right)\right)^{-1} \|\right\} .
$$

This inequality imposes a lower bound on the achievable values of $\gamma$. Furthermore, since $\lambda:=\lambda_{0}-\alpha\left(\lambda_{0}+\mu\right)$, it follows that

$$
\lim _{T_{0} \rightarrow 0, \alpha \rightarrow 0} \frac{e^{\left(\lambda_{0}+\mu\right) T_{0}} \lambda_{0}}{\lambda\left(1-r_{x}\right)^{2}}=\frac{1}{\left(1-r_{x}\right)^{2}} .
$$

The above expression shows that the lower bound on the achievable $\gamma$ in the absence of out of frame events converges to the lower bound derived in [4].

The bound derived in equation (4) is similar to the classical Positional Dilution of Precision (PDOP) metric that is commonly used in navigation systems to determine a lower bound on the achievable error covariance as a function of geometry of the underlying navigation problem $[5,7,8]$. Using our notation, the classical PDOP can be written as

$$
P D O P=\sqrt{\operatorname{tr}\left(H^{T}\left(\mathbf{p}_{C}\right) H\left(\mathbf{p}_{C}\right)\right)^{-1}} .
$$

We therefore see that the new bound derived in this paper captures a worst case performance scenario and the estimate of $x_{C}$ increases the lower bound on the achievable $\gamma$, since

$$
1>\left(1-r_{x}\right)^{2}>0 \text {. }
$$

\section{Experimental setup and flight-test results}

This section describes the experimental setup and the flight test experiments that were performed to test the performance of the nonlinear filter obtained in the previous section. The unmanned air vehicle (UAV) Frog operated by the controls lab at the Naval Postgraduate School (NPS) was equipped with an Infrared video camera. The camera included a Boeing U3000A uncooled 8-12 microns (micrometers). The pixel resolution of the camera was $320 \times 240$. The UAV was also equipped with a Trimble AgGPS 132 Differential Global Positioning System (DGPS). An illustration of the flight test setup is provided 
in Figure 2.

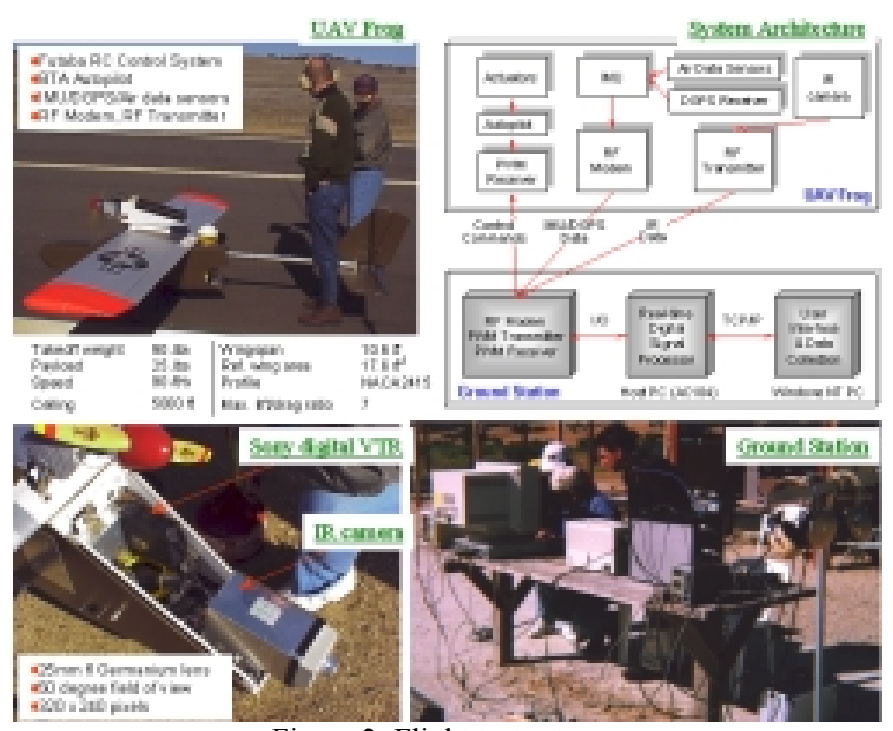

Figure 2: Flight test setup.

Flight tests were conducted at Camp Roberts air field, CA. Charcoal grills were used to model the hot spots on the ship (see Figure 3). Samples of UAV trajectories recorded by onboard DGPS are shown in Figure 4. Similarly, samples of infrared (IR) images collected by the onboard IR camera are shown in Figure 5.

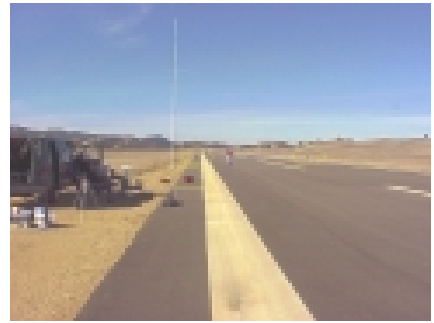
Camp Roberts.

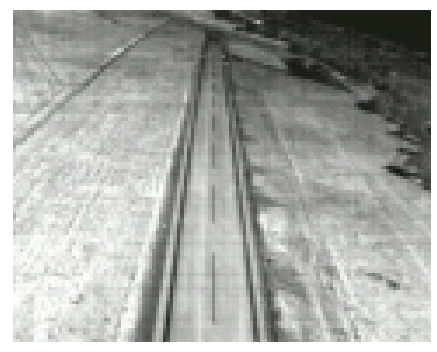

a)
Figure 3: Charcoal grills at

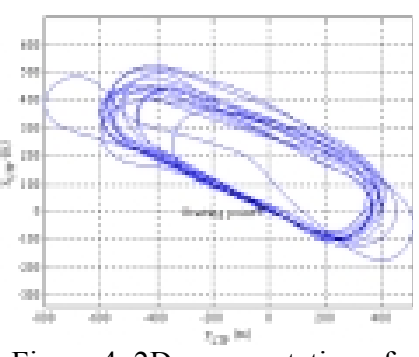

Figure 4: 2D representation of DGPS-recorded trajectories.

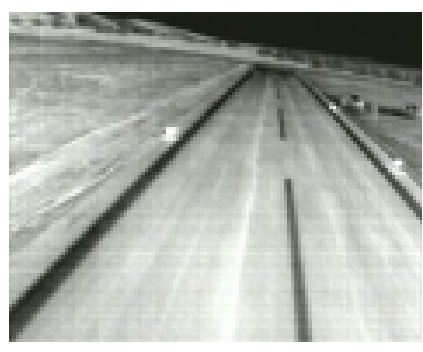

b)
Figure 5: Examples of IR images: a) at the range of $450 \mathrm{~m}$, b) $80 \mathrm{~m}$.

The image plane coordinates and GPS altitude were used by the integrated IR/inertial filter to compute relative position and velocity with respect to the nearest hot spot. Figure 6 shows the results of applying the integrated IR/inertial filter to the flight test data. In particular, the upper graph shows the DGPS landing approach trajectory. The bottom left graph shows the estimation errors computed by comparing the DGPS position with the position estimates produced by the filter. Finally, the bottom right graph shows the response of the filter to an out of frame event. Clearly, the filter performed well.
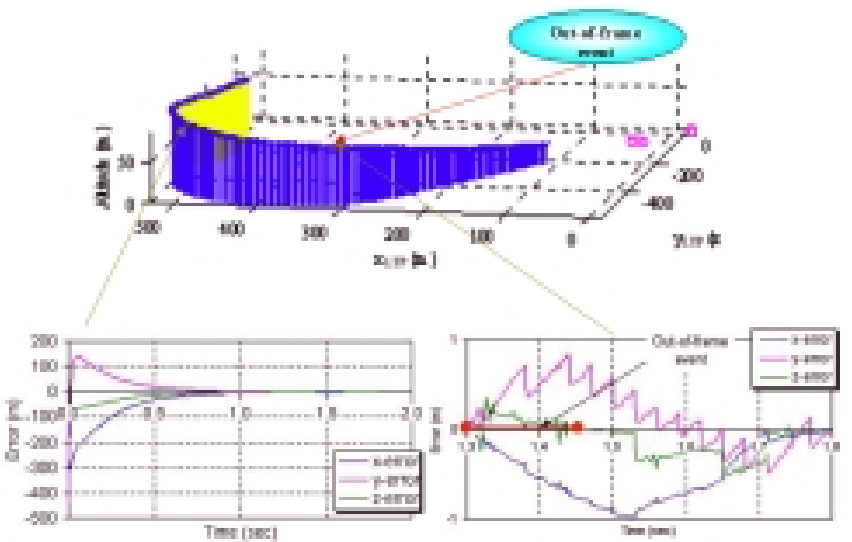

Figure 6: Filter's performance during final approach.

\section{Conclusions}

This paper applied the concept of LPV systems with brief instabilities to the design of an integrated vision/inertial navigation filter with guaranteed stability and performance id the presence of out-of-frame events. Numerical trade-off studies were conducted to determine filter's achievable performance versus the duration of the out-of-frame events. Finally, the filter was tested using flight test data collected by a UAV equipped with inertial sensors and IR camera. The results of the test showed the filter to perform well in the presence of out-of-frame events.

\section{References}

[1] K. Britting. Inertial Navigation System Analysis. WileyInterscience, 1971.

[2] G. Hager, "A Modular System for Robust Positioning using Feedback from Stereo Vision," IEEE Transactions on Robotics and Automation, Vol.13, No.4, August 1997, pp. 582-595.

[3] J. Hespanha, O. Yakimenko, I. Kaminer and A. Pascoal. LPV Systems with Brief Instabilities. NPS Internal Report, 2001.

[4] I. Kaminer, W. Kang, O. Yakimenko and A. Pascoal, "Design of Integrated Vision/Inertial Navigation Systems using Nonlinear Filtering," IEEE Transactions on Aerospace and Electronic Systems, Vol.37, No.1, January 2001, pp. 158-172.

[5] P. Massatt and K. Rundick, "Geometric Formulas for Dilution of Precision Calculations," Journal of Institute of Navigation, Vol.37, No.4, Winter 1990-91, pp. 379-391.

[6] K. Nagpal and P. Khargonekar, "Filtering and Smoothing in an $\mathrm{H}_{\infty}$ setting," IEEE Transactions on Automatic Control, Vol.36, 1991, pp.152-166.

[7] Parikinson and Spilker, Eds. Global Positioning System: Theory and Applications I. Published by AIAA.

[8] G. Siouris. Aerospace Avionics Systems: a Modern Synthesis. Academic Press Inc., 1993. 